\title{
A Correlative Study of Spirometric Parameters and Markers of Right Ventricular Dysfunction in Pediatric Patients with Pulmonary Arterial Hypertension
}

\author{
Carmen Corina Șuteu', lolanda Muntean¹, Cristina Blesneac¹, Brîndușa Căpîlna², Theodora \\ Benedek$^{3}$, Rodica Togănel ${ }^{1}$ \\ 1 Department M4, IIIrd Pediatric Discipline, University of Medicine and Pharmacy, Tîrgu Mureș, Romania \\ 2 Pediatric Clinic, County Emergency Clinical Hospital, Tîrgu Mureș, Romania \\ 3 Department M3, Clinic of Cardiology, University of Medicine and Pharmacy, Tîrgu Mureș, Romania
}

\section{CORRESPONDENCE \\ lolanda Muntean \\ Str. Gheorghe Marinescu nr. 50 \\ 540136 Tîrgu Mureș, Romania \\ Tel: +40744372276 \\ Fax: +40 265210505 \\ E-mail: iolanda.muntean@gmail.com}

\section{ARTICLE HISTORY}

Received: 2 September, 2016 Accepted: 13 September, 2016

Carmen Corina Suteu • Str. Gheorghe Marinescu nr. 50, 540136 Tîrgu Mures, Romania. Tel: +40 744372 276, Fax: +40 265210 505. E-mail: suteucarmen@ yahoo.com

Cristina Blesneac - Str. Gheorghe Marinescu nr. 50 540136 Tîrgu Mureș, Romania. Tel: +40744372276 Fax: +40 265210 505. E-mail: cristinablesneac@ gmail.com

Brîndușa Căpîlna • Str. Gheorghe Marinescu nr. 50 540136 Tîrgu Mureș, Romania. Tel: +40 26521211 E-mail: brindusarc@yahoo.com

Theodora Benedek • Str. Gheorghe Marinescu nr. 38 540139 Tîrgu Mureș, Romania. Tel: +40 265215 551, E-mail: theodora.benedek@gmail.com

Rodica Togănel • Str. Gheorghe Marinescu nr. 50, 540136 Tîrgu Mureș, Romania. Tel: +40 744372 276, Fax: +40 265210 505. E-mail: rodicatoganel@ yahoo.com

\begin{abstract}
Background: Alteration in lung function is present in children with pulmonary arterial hypertension (PAH). We aimed to study the pulmonary function indices obtained by spirometry in pediatric patients with $\mathrm{PAH}$, and to correlate them with B-type natriuretic peptide (BNP) and echocardiographic parameters. Methods: Thirty-one children with PAH were enrolled in this study, of those 26 patients (83.87\%) associated shunt defect and 5 patients (16.12\%) were diagnosed with idiopathic PAH. Forced vital capacity (FVC), forced expiratory volume in one second (FEV1), FEV1/FVC, peak expiratory flow rate (PEFR), forced expiratory flow at 25-75\% of the pulmonary volume (FEF 25-75) were evaluated by spirometry and correlated with BNP and echocardiographic parameters that reflect right ventricle function. Results: Restrictive pattern of pulmonary function was present in $51.6 \%(n=16)$ of all PAH children. There were significant correlations between BNP with FVC ( $p=0.001)$, FEV1 $(p=0.001)$, and FEV1/FVC ( $p=$ 0.001). Serum BNP level was significantly increased in the group of patients without shunt. Of those echocardiographic parameters that reflect right ventricle function, we found that TAPSE significantly correlated with PEFR ( $r=-0.47, p<0.01)$, and with FEF 25-75 ( $r=-0.39, p<0.01)$. Conclusions: Deterioration of the pulmonary function indices are correlated with BNP and echocardiographic parameters, markers of RV dysfunction. Being easy and reliable tests, pulmonary functions can be introduced among the follow-up tools in children with $\mathrm{PAH}$.
\end{abstract}

Keywords: pulmonary function, pulmonary arterial hypertension, children

\section{INTRODUCTION}

Pulmonary arterial hypertension (PAH) is a devastating and progressive disease characterised by vasoconstriction and vascular remodelling of the pulmonary arteries. PAH is currently defined by an mean pulmonary arterial pressure 
(mPAP) $\geq 25 \mathrm{mmHg}$ at rest as assessed by right heart catheterization (RHC), a pulmonary artery wedge pressure (PAWP) $\leq 15 \mathrm{mmHg}$, and a pulmonary vascular resistance (PVR) $>3$ Wood units in the absence of other causes of precapillary $\mathrm{PH} .{ }^{1}$

Pulmonary function tests identify the contribution of underlying airway or parenchymal lung disease. Peacock AJ, considering the proximity of pulmonary vasculature and peripheral airways, postulated that it is possible that the latter may be affected either by the mechanical encroachment of enlarged vessels or by mediators of increased smooth muscle tone or proliferation. ${ }^{2}$ In the context of impaired peripheral airways as a result of pathological mechanisms in the pulmonary vasculature, it is reasonable to postulate that pulmonary function might be affected by the development of $\mathrm{PH} .^{3}$

Assessment of lung function in children is considered an important diagnostic and prognostic tool regarding lung diseases. ${ }^{4}$ Spirometry, the most readily available and useful pulmonary function test, measures the volume of air exhaled at specific time points during a forceful and complete exhalation after a maximal inhalation. The total exhaled volume, known as the forced vital capacity (FVC), the volume exhaled in the first second, known as the forced expiratory volume in one second (FEV1), and their ratio (FEV1/FVC) are the most important variables reported.

In the adult population with IPAH (idiopathic pulmonary arterial hypertension), previous studies of pulmonary function have been contradictory, with normal lung volumes, restrictive ventilator pattern, and airway obstruction. , $^{3,5}$ Pulmonary function tests have not been well-studied in pediatric patients, especially in patients with $\mathrm{PAH}$ associated with congenital heart disease (CHD).

B-type natriuretic peptide (BNP), biomarker of right ventricle (RV) dysfunction, are inversely proportional with prognosis in PAH. ${ }^{6}$ While in the adult pathology there are many data on the prognostic value of BNP serum level, in pediatric PAH the available data are limited, but few records suggest that BNP might be a useful marker in monitoring severity and treatment response assessment. ${ }^{1,7}$ BNP can remain within the normal range despite profund cyanosis from suprasystemic PVR in patients with Eisenmenger syndrome (as long as right heart failure is not present). ${ }^{6}$

Transthoracic echocardiography, due to its noninvasive, safe and more affordable nature, is an important tool for PAH diagnosis and follow-up. ${ }^{7}$ As long as RV performance is the key determinant of exercise capacity and long-term outcomes in patients with $\mathrm{PAH}$, echocardiography remains a highly useful investigation in follow-up, providing quantitative and qualitative information on $\mathrm{RV}$ function with prognostic significance. ${ }^{8}$

The current study included the evaluation of the correlation of spirometric variables with markers of RV in pediatric patients with PAH.

\section{MATERIALS AND METHODS}

This is a prospective, noninterventional study assessing pulmonary function indices, the serum BNP level and echocardiographic parameters that reflect RV function in pediatric patients with PAH. Thirty-one children aged between 4 to 16 years, with a median age of 12 years, were evaluated between December 2013 and January 2015. PAH was defined in accordance with 2015 ESC/ERS Guidelines. ${ }^{1}$ Patients with PAH were distributed into two groups: 1) patients with shunt defect - PAH associated with congenital heart defect (CHD) (group 1, $\mathrm{n}=26$ ) and 2) patients without shunt (IPAH) (group $2, n=5$ ).

Baseline assessment included the WHO (World Health Organization) functional class (FC) and the exercise capacity estimated with 6-minute walk test (6MWT) (in children $\geq 5$ years). The $6 \mathrm{MWT}$ and was performed using standard protocol as ATS guidelines. ${ }^{9}$

\section{Pulmonary assessment}

Spirometry (nSpire 315000 Koko Legend Spirometer, Hertford, UK) was performed using standard technique measuring FEV1, FVC and FEV1/FVC, peak expiratory flow rate (PEFR), forced expiratory flow at $25-75 \%$ of the pulmonary volume (FEF 25-75). The measurement was taken before the echocardiographic examination and was performed using standard protocol as ATS guidelines. ${ }^{10}$ The best trial was obtained from an average of three trials and the predictive values for age, sex, height and weight were calculated. Pulmonary functions obtained were classified as either normal, obstructed airways, restrictive or mixed patterns (Table 1).

\section{Echocardiography}

Conventional 2D and Doppler echocardiography in addition to tissue Doppler imaging were carried out for all pediatric patients with an IE33 equipment (Philips, Amsterndam, Netherlands). The echographic parameters that reflect RV function - tricuspid annular plane systolic excursion (TAPSE), tricuspid annular velocity ( $\left.\mathrm{S}^{\prime}\right)$, fractional area change (FAC), myocardial performance index (Tei), and pericardial effusion were determined by echocardiography. 
TABLE 1. Patterns of pulmonary function in pediatric patients with pulmonary arterial hypertension

\begin{tabular}{|c|c|c|c|c|}
\hline Parameter & Normal & Obstruction & Restriction & Mixed \\
\hline FVC & $80 \%$ of predicted & $\begin{array}{l}\text { Normal or slightly decreased } \\
\text { (70 to } 80 \% \text { of predicted) }\end{array}$ & $\begin{array}{l}\text { Severely decreased } \\
\text { (<70\% of predicted) }\end{array}$ & Decreased \\
\hline \multirow[t]{2}{*}{ FEV1/FVC } & $75-80 \%$ of predicted & $\begin{array}{l}\text { Severely decreased } \\
\text { ( }<60 \% \text { of predicted) }\end{array}$ & $\begin{array}{l}\text { Normal or increased } \\
\text { (>85\% of predicted) }\end{array}$ & Decreased \\
\hline & $n=5(16.1 \%)$ & $n=9(29 \%)$ & $n=16(51.6 \%)$ & $\mathrm{n}=1(3.2 \%)$ \\
\hline
\end{tabular}

FVC - forced vital capacity, FEV1 - forced expiratory volume in one second, FEV1/FVC - forced expiratory volume in one second/ forced vital capacity ratio, $\mathrm{n}$ - number

\section{Measurement of serum BNP level}

The serum samples were collected in each patient in close temporal proximity to the echocardiographic assessment. Serum BNP (range $0-100 \mathrm{pg} / \mathrm{mL}$ ) was measured by an ARCHITECT i 1000SR Immunoassay Analyzer equipment (Abbott Diagnostics, Illinois, USA) in peripheral venous blood samples.

The study protocol conforms to the ethical guidelines of the 1975 Declaration of Helsinki, and the informed consent was taken from all parents before taking any data or doing any tests.

\section{Statistical analysis}

SPSS software (Statistical Package for Social Sciences, 20 version, Chicago, IL, USA) was applied for statistical analysis. Data were labeled as binary or quantitative nominal variables. Nominal variables were performed by frequency tables and chi-square statistical test was applied. The quantitative variables were tested using Kolmogorov-Smirnov test and were described as mean \pm standard deviation, or median and percentile (25\%, $75 \%)$. Differences between means or medians were analyzed using paired Student's t test or the Mann-Whitney test. The statistically significant level was set to a value of $\mathrm{p}<0.05$.

\section{RESULTS}

The study included 31 children ( 13 girls $-41.9 \%$, 18 boys - 58.06\%), the median age was 12 years, and the average weight was $32.1 \mathrm{~kg}$.
The result of pulmonary function tests of the studied patients are shown in Table 1. Restrictive pattern of functions was present in $51.6 \%(n=16)$ of all PAH children, followed by obstructive pattern $(n=9.29 \%)$.

The correlation of BNP with the pulmonary function indices in the studied patients documented a significant association of BNP with FVC $(p=0.001)$, FEV1 $(p=0.001)$, and FEV1/FVC ( $\mathrm{p}=0.001)$ (Table 2).

When we studied the correlation between echocardiographic parameters that reflect RV function and the pulmonary function indices in all patients included in the study, we found that there was a significant correlation between TAPSE and PEFR $(\mathrm{r}=-0.47, \mathrm{p}<0.01)$, and TAPSE and FEF 25-75 $(\mathrm{r}=-0.39, \mathrm{p}<0.01)$ (Table 3$)$.

Of the 31 pediatric patients with PAH included in the study, 26 patients (83.87\%) associated shunt defect (group 1 ), and 5 patients (16.12\%) were diagnosed with IPAH (group 2). In our study we could not find any significant differences in regard to the age, sex, and weight between group 1 and group 2, but there was a significant difference between these two groups regarding the length $(p=0.04)$ (Table 4).

We could not find any significant associations between the functional parameters in the two studied groups: functional class $(\mathrm{p}=0.38)$ and $6 \mathrm{MWT}(\mathrm{p}=0.35)$, respectively (Table 4). There were no statistically significant differences between children with shunt defect and children without shunt defect regarding the results of pulmonary function tests ( $p>0.05$ ) (Table 4). Serum BNP level was significantly increased in the group of patients without shunt (group 2) $(\mathrm{p}=0.04)($ Table 4$)$.

Of the echocardiographic parameters that reflect RV function there was a significant difference between groups

TABLE 2. Correlation of pulmonary function indices with B-type natriuretic peptide

\begin{tabular}{cccccc}
\hline & FVC & FEV1 & FEV1/FVC & PEFR & FEF 25-75 \\
\hline BNP & $p=0.001$ & $p=0.001$ & $p=0.001$ & $p=0.244$ & $p=0.123$ \\
\hline
\end{tabular}

FVC - forced vital capacity, FEV1 - forced expiratory volume in one second, FEV1/FVC - forced expiratory volume in one second/ forced vital capacity ratio, PEFR - peak expiratory flow rate, FEF $25-75$ - forced expiratory flow at $25-75 \%$ of the pulmonary volume, BNP - B-type natriuretic peptide 
TABLE 3. Correlation of pulmonary function indices with echocardiographic parameters that reflect right ventricular function

\begin{tabular}{lccccc}
\hline $\begin{array}{l}\text { Echo parameters that reflect } \\
\text { RV function }\end{array}$ & FVC & FEV1 & FEV1/FVC & PEFR & FEF 25-75 \\
\hline TAPSE & $r=-0.04$ & $r=-0.15$ & $r=-0.005$ & $r=-0.47^{*}$ & $r=-0.39^{*}$ \\
FAC & $r=0.11$ & $r=0.13$ & $r=0.32$ & $r=0.002$ & $r=0.09$ \\
Tei & $r=-0.10$ & $r=-0.07$ & $r=0.11$ & $r=0.06$ & $r=0.08$ \\
$S^{\prime}$ & $r=-0.08$ & $r=-0.20$ & $r=-0.34$ & $r=-0.26$ & $r=-0.19$ \\
pericarditis & $66.5 \pm 9.9$ & $67.3 \pm 6.9$ & $105.9 \pm 4.8$ & $56.1 \pm 16.1$ & $76.5 \pm 16.8$ \\
\hline
\end{tabular}

FVC - forced vital capacity, FEV1 - forced expiratory volume in one second, FEV1/FVC - forced expiratory volume in one second/forced vital capacity ratio, PEFR - peak expiratory flow rate, FEF $25-75$ - forced expiratory flow at $25-75 \%$ of the pulmonary volume, TAPSE - tricuspid annular plane systolic excursion, FAC - fractional area change, Tei - myocardial performance index of right ventricle, $\mathrm{S}$ - tricuspid annular velocity

$*$ - very significant correlation $(\mathrm{p}<0.01)$

Pearson Correlation -r

in FAC $(\mathrm{p}=0.04)$. Interestingly, no differences regarding TAPSE, Tei, $\mathrm{S}^{\prime}$, and pericardial effusion were found in the study groups.

\section{DISCUSSION}

Pulmonary function tests identify the contribution of underlying airway or parenchymal lung disease. The results of the study showed that pulmonary function tests are modi- fied in pediatric patients with PAH. Leonard $\mathrm{M}$ et al. found that children with severe $\mathrm{PAH}$ associated with CHD presented significant reduction in FVC. ${ }^{11}$ Our study confirmed that restrictive pattern is the most striking characteristic of pulmonary function test, followed by the obstructive pattern. These findings are in agreement with the study of Sabry AF et al. ${ }^{4}$ The restrictive pattern, characterized by severely decreased FVC, is due to the lung stiffness and to the reduced lung compliance secondary to increased pul-

TABLE 4. Comparison of baseline clinical, functional, laboratory, pulmonary function indices and echocardiographic parameters characteristics between $\mathrm{PAH}$ pediatric groups

\begin{tabular}{lcccc}
\hline & $\begin{array}{c}\text { All pediatric } \\
\text { patients with PAH } \\
(\mathbf{n}=\mathbf{3 1})\end{array}$ & $\begin{array}{c}\text { Group 1 } \\
\text { Shunt defect } \\
\mathbf{( n = 2 6 )}\end{array}$ & $\begin{array}{c}\text { Group 2 } \\
\text { No shunt defect } \\
\mathbf{( n = 5 )}\end{array}$ & p value \\
\hline Age, yr & $12(4-16)$ & $12.5(4-16)$ & $9(6-12)$ & $0.11^{* *}$ \\
Female gender & $13(41.9)$ & $10(38.5)$ & $3(60.0)$ & $0.37 \#$ \\
Weight, kg & $32.1 \pm 13.9$ & $33.1 \pm 14.1$ & $26.8 \pm 7.4$ & $0.33^{*}$ \\
Length, cm & $138 \pm 20.1$ & $140.2 \pm 20.8$ & $126.4 \pm 10.5$ & $0.04^{*}$ \\
WHO FC II/II/IV & $17 / 12 / 2$ & $15 / 10 / 1$ & $2 / 2 / 1$ & $0.38 \#$ \\
6MWT distance, m & $394.1 \pm 12.39$ & $403.1 \pm 10.5$ & $348.0 \pm 18.3$ & $0.35^{*}$ \\
BNP, pg/ml & $49.7(4.2-192.5)$ & $32.2(4.2-180.7)$ & $77.7(15.8-192.5)$ & $0.04^{* *}$ \\
FVC & $67.8 \pm 15.3$ & $67.5 \pm 16.1$ & $69.4 \pm 11.5$ & $0.81^{*}$ \\
FEV1 & $67.8 \pm 14.9$ & $66.7 \pm 15.4$ & $72.2 \pm 8.2$ & $0.45^{*}$ \\
FEV1/FVC & $106.5 \pm 7.3$ & $106.7 \pm 7.6$ & $105.8 \pm 6.5$ & $0.79^{*}$ \\
PEFR & $58.8 \pm 16.2$ & $58.3 \pm 16.8$ & $61.4 \pm 13.5$ & $0.70^{*}$ \\
FEF 25-75 & $78.9 \pm 26.2$ & $77.7 \pm 27.1$ & $85.2 \pm 21.9$ & $0.56^{*}$ \\
Echo parameters that reflect RV function & & & \\
TAPSE & $18.9 \pm 5.3$ & $19.1 \pm 5.3$ & $17.9 \pm 6.2$ & $0.65^{*}$ \\
FAC & $36.2 \pm 9.2$ & $37.8 \pm 8.6$ & $28.8 \pm 8.6$ & $0.04^{*}$ \\
Tei & $0.65 \pm 0.17$ & $0.63 \pm 0.17$ & $0.73 \pm 0.14$ & $0.27^{*}$ \\
S & $10.1 \pm 2.6$ & $10.1 \pm 2.8$ & $10.4 \pm 2.1$ & $0.72^{*}$ \\
pericarditis & $7(23.3)$ & $5(20.0)$ & $2(40.0)$ & $0.33^{*}$ \\
\hline
\end{tabular}

PAH - pulmonary arterial hypertension, WHO FC - functional class, 6MWT - six minute walk test, BNP - B-type natriuretic peptide, FVC - forced vital capacity, FEV1 - forced expiratory volume in one second, FVC/FEV1 - forced vital capacity/forced expiratory volume in one second ratio, PEFR - peak expiratory flow rate, FEF $25-75$ - forced expiratory flow at $25-75 \%$ of the pulmonary volume, TAPSE - tricuspid annular plane systolic excursion, FAC fractional area change, Tei - myocardial performance index of right ventricle, $S^{\prime}$ - tricuspid annular velocity

${ }^{*} p$ value using Student's t-test; ${ }^{* *} p$ value using Mann Whitney test; \#p value using Chi-square test 
monary pressure. Abnormalities in endothelial function, including impaired production of prostacyclin and nitric oxide, and excessive synthesis of endothelin have wellknown effects on the bronchial system.,12 Martin C et al., examining the effect of the endothelin receptor antagonist bosentan on endothelin-induced bronchoconstriction, concluded that bosentan completely prevents ET-receptor agonist (IRL1620)-induced bronchoconstriction. ${ }^{13}$ In the evolution of PAH patients, cardiac enlargement with its compressing effect and left ventricular dysfunction cause impaired respiratory muscle perfusion, which add an obstructed element. ${ }^{4}$

The ability to walk for a distance is an easy way to measure exercise capacity in patients with cardiac and pulmonary disease. Although some data are available, normative values of 6MWT for age, sex, and leg length have not been well standardized in children, and the predictive value of the $6 \mathrm{MWT}$ in pediatric PAH has not been proven. ${ }^{6,14} \mathrm{In}$ many centers the changes in $6 \mathrm{MWT}$ are used for longitudinal monitoring of patients. In our study and in another study done by Jing ZC et al. it was found that no functional parameters correlated well with pulmonary function indices. ${ }^{3}$ Significant correlation between 6MWT and FEV1 has been shown in other studies involving patients with chronic pulmonary disease. ${ }^{15}$

Considering all PAH patients, the present study showed that FVC, FEV1, FEV1/FVC had very significant positive correlation with serum BNP level, marker of the RV dysfunction. Bernus A et al. found that BNP correlated with hemodynamic parameters, which have been shown to be predictive of outcomes in $\mathrm{PAH}$, and are considered indicators for disease severity. In addition, they observed that a high BNP is indicative of poor survival, more so in IPAH patients. ${ }^{16}$ Comparing the two studied groups, the current study could demonstrate significant differences regarding the BNP, elevated levels of BNP being revealed in pediatric patients with IPAH. The current study showed that pulmonary function indices are associated with RV dysfunction, which is more present in patients with IPAH.

As long as RV function is an important determinant of clinical status and outcomes in PAH, the assessment of RV function by echocardiography continues to play a central role in PAH diagnosis and follow-up. ${ }^{6}$ Echocardiography can measure a number of RV parameters that have been shown to be key determinants in the prognosis of PAH, and which may be used as specific goals for treatment. Recently, TAPSE, alongside FC and NT-proBNP (N-terminal pro b-type natriuretic peptide), has been recognized as treatment goal in pediatric PAH. ${ }^{8}$ The results in this study show that PEFR and FEF 25-75, indicating small airway obstruction pattern, correlate with TAPSE. Some published studies have evaluated the correlation between spirometric parameters and pulmonary artery systolic pressure estimated by echocardiography. ${ }^{18,19}$ However, from our knowledge, no study has evaluated the correlation of pulmonary function indices with echocardiographic parameters that reflect RV function, until now.

The main limitation of our present work is that it was an observational study with a small number of pediatric patients diagnosed with PAH. In addition, the studied group is heterogeneous.

\section{CONCLUSION}

The positive correlations of pulmonary function indices with BNP and with echocardiographic parameters that reflect RV function support the idea that resting lung function measurements can be introduced among the follow-up tools in children with PAH. The restrictive pattern was associated with most of the patients of the study, proving that lung compliance is affected in pediatric patients with PAH.

\section{CONFLICT OF INTEREST}

Nothing to declare.

\section{REFERENCES}

1. Gallie N, Humbert M, Vachiery JL, et al. 2015 ESC/ERS Guidelines for the diagnosis and treatment of pulmonary hypertension. Eur Heart J. 2016;37:67-119

2. Peacock AJ. Primary pulmonary hypertension. Thorax. 1999;54:1107-1118

3. Jing ZC, Xu XQ, Badesch DB, et al. Pulmonary function testing in patients with pulmonary arterial hypertension. Respir Med. 2009;103:1136-1142.

4. Sabry AF, El-Hagrasi HF, Hossam H, et al. Changes in pulmonary functions in children in responses to pulmonary hypertension associated with cardiac disease in Suez Canal Area in Egypt. Med J Cairo Univ. 2013;81(2):101-106

5. Meyer FJ, Ewert R, Hoeper MM, et al. Peripheral airway obstruction in primary pulmonary hypertension. Thorax. 2002;57:473-476.

6. Abman SH, Hansmann G, Archer SL, et al. Pediatric Pulmonary Hypertension Guidelines from the American Heart Association and American Thoracic Society. Circulation. 2015;132(21):2037-2099.

7. Colvin KL, Dufva MJ, Delaney RP, Ivy DD, Stenmark KR, Yeager ME. Biomarkers for pediatric pulmonary arterial hypertension - a call to collaborate. Front Pediatr. 2014;2(7):1-12.

8. Ploegstra MJ, Douwes J, Roofthooft MTR, Zijlstra WM, Hillege HL, Berger RM. Identification of treatment goals in paediatric pulmonary arterial hypertension. Eur Respir J. 2014;44:1616-1626.

9. American Thoracic Society. American Thoracic Society statement: guidelines for the six-minute walk test. Am J Respir Crit Care Med. 2002;166:111-117

10. Laszlo G. Standardisation of lung function testing: helpful guidance from the ATS/ERS Task Force. Thorax. 2006;61(9):744-746.

11. Linde LM, Siegel SI, Martelle RR, Simmons DH. Lung function in congenital heart disease. Chest. 1964;46(1):46-50

12. Giaid A, Saleh D. Redeced expression of endothelin nitric oxide synthase in the lungs of patients with pulmonary hypertension. $N$ Engl J Med. 1995;333(4):214-221.

13. Martin C, Held HD, Uhlig S. Differential effects of the mixed ET(A)/ 
ET(B)-receptor antagonists bosentan on endothelin-induced bronchoconstriction, vasoconstriction and prostacyclin release. Naunyn Schmiedebergs Arch Pharmacol. 2000;362:128-136.

14. Lammers AE, Hislop AA, Flynn Y, et al. The 6-minute walk test: normal values for children of 4-11 years of age. Arch Dis Child. 2008;93:464-468.

15. Nixon PA, Joswiak ML, Fricker FJ. A six-minute walk test for assessing exercise tolerance in severely ill children. J Pediatr. 1996;129:362-366.

16. Bernus A, Wagner BD, Accurso F, et al. Brain Natriuretic Peptide Levels in Managing Pediatric Patients with Pulmonary Arterial Hypertension. Chest. 2009;135(3):745-751.
17. D'Alonzo GE, Barst RJ, Ayres SM, et al. Survival in patients with primary pulmonary hypertension. Results from a national prospective registry. Ann Intern Med. 1991;115:343-349.

18. Sultan K, Hussain M, Ismael A. The relation of echocardiographic findings to pulmonary function tests in patients with chronic obstructive pulmonary disease. J Fac Med Baghdad. 2009;51(1):17-22.

19. Higham MA, Dawson D, Joshi J, et al. Utility of echocardiography in assessment of pulmonary hypertension secondary to COPD. Eur Respir J. 2001;17:350-355 